\title{
Structure and Saccharification of Sugarcane Bagasse Pretreated with Acid Coupled Alkaline
}

\author{
Lihong Cui*, Xiaoyi Wei, Jihua Li, Gang Chang, \\ Xiaobing Huang
}

Key Laboratory of Tropical Crop Products Processing of Ministry of Agriculture,

Agriculture Products Processing Research Institute, Chinese Academy of Tropical Agricultural Sciences, Zhanjiang

\author{
524001, China \\ LH_cui@126.com
}

\begin{abstract}
Effect of acid coupled alkaline pretreatment on structure and enzymatic saccharification of sugarcane was investigated. The original and pretreated sugarcane bagasse samples were thoroughly characterized by Fourier transform infrared spectroscopy (FT-IR), X-ray diffraction (XRD) and scanning electron microscopy (SEM). And the composition of sugarcane bagasse was investigated by lignocellulose determination. It was found that acid coupled alkaline pretreatment could effectively remove the hemicellulose and lignin from sugarcane bagasse, and the mass fraction of cellulose increased from $54.43 \%$ to $93.53 \%$. The original sample experienced an increase in the content of the free hydroxyl group and surface area during the pretreatment, whereas the diffraction typical of cellulose had no change. The results suggested that this pretreatment led to effective disruption of sugarcane bagasse for subsequent enzyme hydrolysis as evidenced by a high saccharification rate of $77.86 \%$ within $30 \mathrm{~h}$.
\end{abstract}

Keywords-Sugarcane bagasse, Pretreatment, Cellulose, Enzymatic hydrolysis, Acid Coupled Alkaline Pretreatment

\section{INTRODUCTION}

Lignocellulose, whether in the form of dedicated energy crops (e.g. switchgrass), agricultural or forestry residues (e.g. sugarcane bagasse), present an abundant renewable energy resource [1]. Along with the decreasing reserves of fossil fuel resources and the increasing of world energy demand, the utilization of biomass as renewable and sustainable feedstock to produce alternative fuels and chemicals have attracted much interest in recent years. Cellulose is the most abundant component in lignocellulose and has a great potential in the conversion into bio-fuels, chemicals and materials [2]. But these polysaccharide molecules are difficult to hydrolyze into fermentable sugars since they are contained in the chemically recalcitrant and structurally complex lignocellulosic matrix. Therefore, a pretreatment step is required in order to increase the material digestibility for the subsequent enzymatic hydrolysis. Various pretreatment methods have been reported to remove lignin and hemicellulose, reduce cellulose crystallinity, and change chemical groups, for instance, milling, steam explosion,

The authors are extremely grateful for the financial support from National Nonprofit Institute Research Grant of CATAS (No.1630062013012 \& No.1630122017018) and Zhanjiang agricultural research project (No. 2016A03019).

\author{
Donghui Han \\ College of food science and technology \\ Huazhong Agricultural University \\ Wuhan 430070, China
}

dilute acid, liquid hot water, dilute lye, wet oxidation, and ammonia fiber explosion (AFEX) [3, 4].

In the present study, an effective lignocellulosic biomass pretreatment process was developed using $3 \mathrm{wt} \%$ nitric acid and $1.5 \mathrm{wt} \%$ sodium hydrate under a mild condition. The changes of the structure and enzymatic hydrolysis of sugarcane bagasse were investigated. The structural modifications of sugarcane bagasse were studied by Fourier transform infrared spectroscopy (FT-IR), X-ray diffraction (XRD) and scanning electron microscopy (SEM). Saccharification rate of treated sugarcane bagasse cellulose was also compared with the original feedstock.

\section{EXPERIMENTAL SECTION}

\section{A. Materials}

Sugarcane bagasse was obtained from a local sugar factory (Zhanjiang, China). It was air-dried, milled, screened to obtain the particles of 40-80 mesh, and then stored until used. Cellulase from Aspergillus niger was purchased from Sukahan Bio-tech Co., Ltd. All other reagents and solvents were of analytical grade.

\section{B. Pretreatment}

The sugarcane bagasse powder was soaked in a round-bottom flask at room temperature in $3 \mathrm{wt} \%$ nitric acid with a solid to liquor ratio of 1:15. The flask was then placed in a boiling water bath for $3 \mathrm{~h}$. The acidic slurry was filtered through Whatman filter paper with a Buchner funnel. The recovered solids were washed with deionized water until neutrality and then mixed with the same volume of $1.5 \mathrm{wt} \%$ sodium hydrate. After then the mixture was placed in boiling bath for $1.5 \mathrm{~h}$. Finally, the solid fraction (pretreated bagasse) was washed with deionized water until neutrality and dried in vacuum freeze drying equipment.

\section{FT-IR Spectroscopy}

FT-IR spectra of the original and pretreated samples were recorded using a Fourier Transform Infrared Spectrometer 
(Spectrum GX-1, PerkinElmer, USA). The samples were dried at $80^{\circ} \mathrm{C}$ under vacuum for $12 \mathrm{~h}$ before analysis. Then a small quantity (approximately $1 \mathrm{mg}$ ) samples were blended with 300 $\mathrm{mg}$ of $\mathrm{KBr}$ powder and compressed to form a disc. The spectra for each sample were recorded as an average of 100 scans at a resolution of $1 \mathrm{~cm}^{-1}$ in the range from 4000 to $400 \mathrm{~cm}^{-1}$.

\section{Crystalline Analyzed by XRD}

The XRD pattern of the original and pretreated samples was measured using a Bruker D8 Advance diffractometer with a $\mathrm{Cu}$ $\mathrm{K} \alpha$ radiation $(\lambda=0.154 \mathrm{~nm})$ at $40 \mathrm{kV}$ and $20 \mathrm{~mA}$. The sample was scanned and the recorded intensity in $2 \theta$ ranged from $5^{\circ}$ to $80^{\circ}$

\section{E. Surface Morphology Observation by SEM}

The morphology analysis of the original and pretreated sugarcane bagasse was done by scanning electron microscopy (SEM). The SEM pictures were taken at different magnifications using environmental scanning electron microscope model QUANTA250 (FEI, US) with an accelerating voltage of $20 \mathrm{kV}$. The samples were coated with 20 $\mathrm{nm}$ of gold and kept in a desiccator until analysis.

\section{F. Determination of Sugarcane Bagasse Composition}

The composition of the original and pretreated sugarcane bagasse samples was determined according to Van Soest method [5].

\section{G. Enzymatic Hydrolysis}

Batch enzymatic hydrolysis of untreated and pretreated sugarcane bagasse samples was carried out at $50^{\circ} \mathrm{C}$ in a reciprocating shaker bath for $30 \mathrm{~h}$. All samples were diluted to $10 \mathrm{~g} / \mathrm{L}$ in a $0.05 \mathrm{M}$ citrate buffer with $\mathrm{pH} 4.8$ for enzymatic hydrolysis. Cellulase activity was 180 FPU per gram of original enzyme power, which determined using standard protocol from the National Renewable Energy Laboratory (NREL) (www.nrel.org). The cellulase concentration of 14 FPU per gram substrate. The reaction was monitored by withdrawing samples from the supernatant periodically, and measuring the released reducing sugars by the DNS assay using D-glucose as a standard [6].

Saccharification rate from untreated and pretreated sugarcane bagasse samples was calculated according to the literature [3] as follows:

$$
\mathrm{SR}(\%)=\frac{R G}{P S} \times 100
$$

where RG is the dry-weight of reducing sugars in enzyme hydrolysis supernatant, PS is the dry-weight sugarcane bagasse in pretreated solids.

\section{RESULTS AND DISCUSSION}

\section{A. FT-IR Spectra}

Fig. 1 illustrates the FT-IR spectra of the original and pretreated sugarcane bagasse. The peak at $\sim 1510 \mathrm{~cm}^{-1}$ is attributed to aromatic skeletal vibrations of lignin $[7,8]$. The absorption at $\sim 1730 \mathrm{~cm}-1$ is due to carbonyl groups of uronic acid from hemicellulose [9]. Compared with the spectra of original sugarcane bagasse (Fig. 1a), it can be observed that absorption peaks at $1732 \mathrm{~cm}^{-1}, 1513 \mathrm{~cm}^{-1}$ and $1632 \mathrm{~cm}^{-1}$ disappear in the spectra of the acid coupled alkaline pretreated sample (Fig. 1b). This implied that the removal of hemicellulose and lignin from sugarcane bagasse by acid coupled alkaline pretreatment. The absorption of $\mathrm{O}-\mathrm{H}$ stretching vibration shift from $3340 \mathrm{~cm}^{-1}$ of the original sample to $3384 \mathrm{~cm}^{-1}$ of the pretreated sugarcane bagasse, indicating the content of the free hydroxyl group in sugarcane bagasse increased after pretreatment [2].

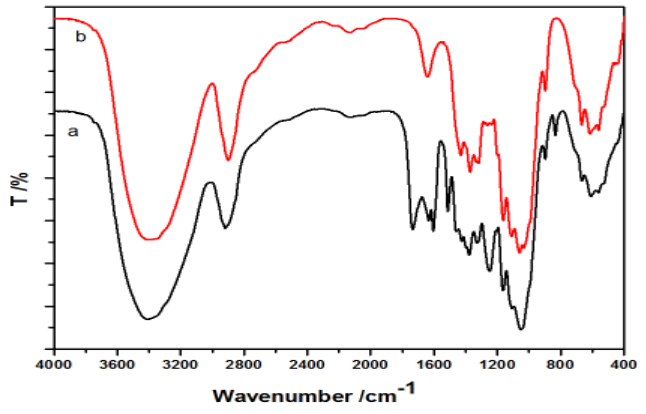

Fig. 1. FT-IR spectra of original (a) and pretreated sugarcane bagasse (b).

\section{B. X-Ray Pattern}

XRD patterns of original and pretreated sugarcane sample were shown in Fig. 2. The diffractograms of both the original and pretreated sample exhibit diffraction typical of cellulose I, with diffractogram peaks at $14.7^{\circ}, 16.4^{\circ}$ and $22.6^{\circ}[10]$. Based on the XRD patterns, the crystallinity index of original and pretreated sugarcane bagasse were calculated by using the empirical method of Segal [11]. After acid coupled alkaline pretreatment, the crystallinity of sugarcane bagasse cellulose was $58.34 \%$, which was $35 \%$ higher than that of original sample. The increase of cellulose crystallinity was due to the significant increase of cellulose content in pretreated bagasse.

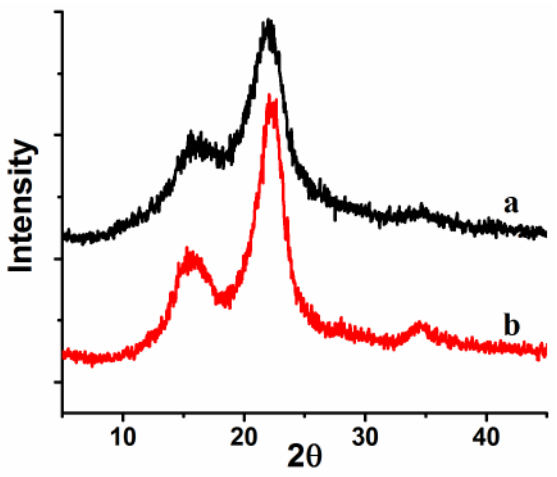

Fig. 2. XRD patterns of original (a) and pretreated sugarcane bagasse cellulose (b).

\section{Surface Morphology Observation}

SEM was a useful tool to investigate the surface structure of lignocellulosic biomass. Fig. 3 shows SEM images at 1500× 
magnifications of the original and pretreated sugarcane bagasse. The original sugarcane bagasse has a tight structure with some pores on the surface. Compare to the untreated samples, acid coupled alkaline pretreatment significantly alters the surface structure of sugarcane bagasse. It can be attributed to the almost completely removal of hemicellulose and lignin in sugarcane bagasse by acid coupled alkaline pretreatment, thus interrupted the crosslinking between cellulose, hemicellulose and lignin. This morphological structure could suggest alarge accessible surface to the cellulase, which was in agreement with the reducing sugar yield obtained.
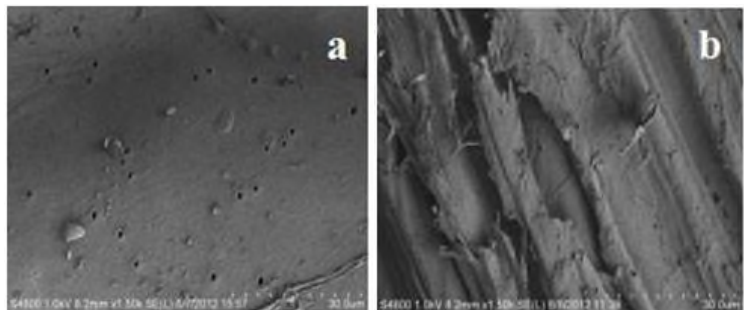

Fig. 3. SEM images of original (a) and pretreated sugarcane bagasse (b).

\section{Determination of Sugarcane Bagasse Composition}

The effect of acid coupled alkaline pretreatment on the composition of sugarcane bagasse was shown in Fig. 4. When compared with the untreated sample, the content of hemicellulose and lignin were almost clearly removed with the acid coupled alkaline pretreatment. This result was consistent with that of the FT-IR spectra. Since hemicellulose and lignin is a physical barrier that surrounds cellulose fibers and protect them from enzymatic attack [12], their removal indicates the potential of the used method for activation of bagasse for enzymatic hydrolysis. After treated by acid coupled alkaline, the content of cellulose in sugarcane bagasse increased sharply from $54.43 \%$ to $93.53 \%$.

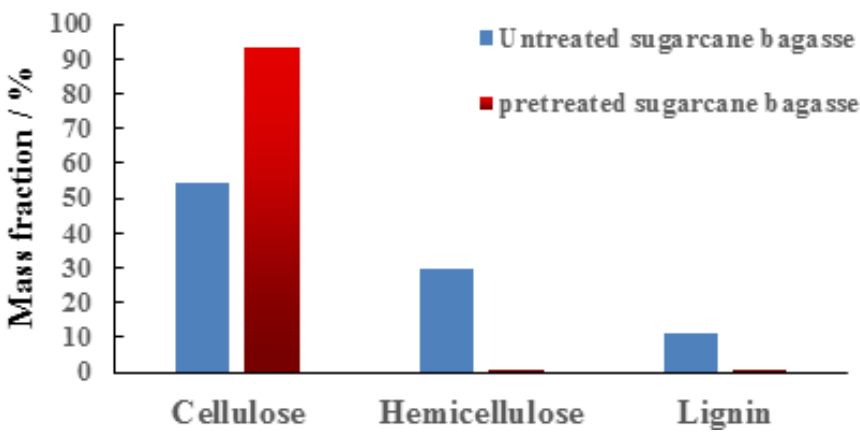

Fig. 4. Composition of original and pretreated sugarcane bagasse.

\section{E. Enzymatic Hydrolysis}

Fig. 5 shows the time course of enzymatic hydrolysis. As can be seen, the conversion of both the original and pretreated sugarcane bagasse to reducing sugar increased with the increase of the hydrolysis time. But it was found that the enzymatic saccharification rate of sugarcane bagasse was increased after pretreated with acid coupled alkaline. Saccharification rate of pretreated sugarcane bagasse reached $77.86 \%$ within $30 \mathrm{~h}$, whereas untreated sample only reached $5.23 \%$ over the same time interval. The results suggested that acid coupled alkaline pretreatment provided an effective process to enhance the conversion of cellulose to glucose. The enhancement would mainly be ascribed to the structural changes as determined in the present study, such as an increase in the free hydroxyl group and the surface area of the cellulose.

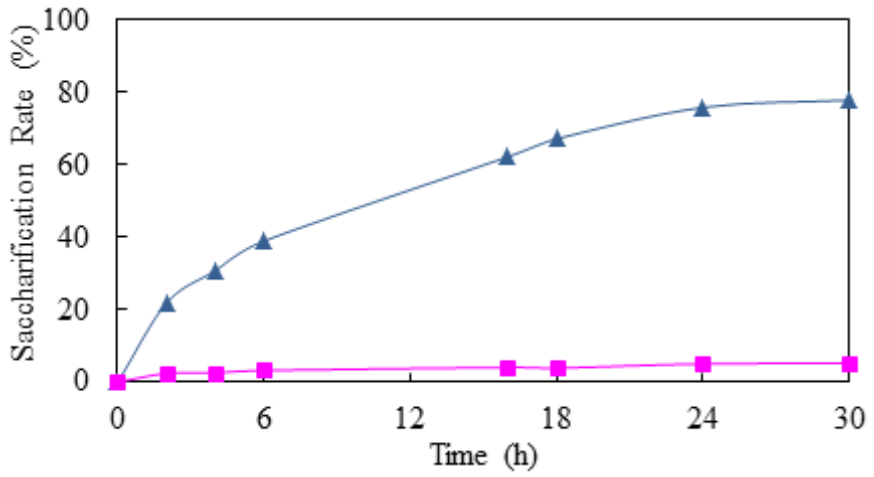

Fig. 5. Enzymatic hydrolysis of original (-ח-) and pretreated (- $\left.\boldsymbol{\Delta}_{-}\right)$sugarcane bagasse.

\section{CONCLUSIONS}

Acid coupled alkaline pretreatment of sugarcane bagasse was investigated in this work. The structure of sugarcane bagasse was significantly changed after pretreatment, such as the removal of hemicellulose and lignin, an increase in cellulose content, and more free hydroxyl group was exposed. The enzymatic saccharification rate of pretreated sugarcane bagasse cellulose was largely enhanced, indicating that the structural modifications of sugarcane bagasse are significant in relation to the enzymatic hydrolysis. Therefore, acid coupled alkaline treatment is a promising pretreatment process for lignocellulose biomass in a commercial biorefinery.

\section{REFERENCES}

[1] S.K. Karatzos, L.A. Edye, W.O. S. "Doherty. Sugarcane bagasse pretreatment using three imidazolium-based ionic liquids; mass balances and enzyme kinetics". Biotech. Biofuel. vol. 5, no. 1, pp. 62, 2012.

[2] J. Bian, F. Peng, X.P. Peng, et al. "Effect of [Emim]Ac pretreatment on the structure and enzymatic hydrolysis of sugarcane bagasse cellulose". Carbohydrate Polym. vol. 100, pp. 211-217, 2014.

[3] F.H. Li, H. Hu, R.S. Yao, et al. "Structure and saccharification of rice straw pretreated with microwave-assisted dilute lye". Ind. Eng. Chem. Res. vol. 51, no. 17, pp. 6270-6274, 2012.

[4] Y. Sun, J.J. Cheng. "Dilute acid pretreatment of rye straw and bermudagrass for ethanol production". Biores. Tech. vol. 96, no. 14, pp. 1599-1606, 2005

[5] P.J. Van Soest, J.B. Robertson, B.A. Lewis. "Methods for dietary fiber, neutral detergent fiber, and nonstarch polysaccharides in relation to animal nutrition”. J. Dairy Sci. vol. 74, no. 10, pp. 3583-3597, 1991.

[6] G.L. Miller. "Use of dinitrosalicylic acid reagent for determination of reducing sugar". Anal. Chem. vol. 31, pp. 426-442, 1959.

[7] X. Colom, F. Carrillo, F. Nogués, et al. "Structural analysis of photodegraded wood by means of FTIR spectroscopy". Polym. Degradat. Stabil. vol. 80, no. 3, pp. 543-549, 2003.

[8] M. Schwanninger, J.C. Rodrigues, H. Pereira, et al. "Effects of short-time vibratory ball milling on the shape of FT-IR spectra of wood and cellulose". Vibrat. Spectr. vol. 36, no. 1, pp. 23-40, 2004.

[9] A.M. Adel, Z.H. A. El-Wahab, A.A. Ibrahim, et al. "Characterization of microcrystalline cellulose prepared from lignocellulosic materials. Part I. 
Acid catalyzed hydrolysis”. Biores. Tech. vol. 101, no. 12, pp. 4446-4455, 2010.

[10] A.E. Oudiani, Y. Chaabouni, S. Msahli, F. Sakli. "Crystal transition from cellulose I to cellulose II in $\mathrm{NaOH}$ treated Agave americana L. fibre". Carbohydrate Polym. vol. 86, no. 3, pp. 1221-1229, 2011.

[11] L. Segal, L. Creely, A.E. Martin. "An empirical method for estimating the degree of crystallinity of native cellulose using the X-ray diffractometer". Text. Res. J. vol. 29, pp. 786-794, 1959.

[12] M.J. Taherzadeh, K. Karimi. "Pretreatment of lignocellulosic wastes to improve ethanol and biogas production: a review". Int. J. Molecul. Sci. vol. 9, pp. 1621-1651, 2008 . 